\title{
TFAP2C facilitates somatic cell reprogramming by inhibiting c-Myc-dependent apoptosis and promoting mesenchymal-to-epithelial transition
}

\author{
Yuan Wang', Shuang Chen', Qingyuan Jiang ${ }^{1,2}$, Jie Deng ${ }^{1}$, Fuyi Cheng ${ }^{1}$, Yi Lin' ${ }^{1}$ Lin Cheng ${ }^{1}$, Yixin Ye ${ }^{1}$, Xiaolei Chen ${ }^{1}$,
} Yunqi Yao ${ }^{1}$, Xiaomei Zhang ${ }^{1,3}$, Gang Shi ${ }^{1}$, Lei Dai ${ }^{1}$, Xiaolan $\mathrm{Su}^{1}$, Yong Peng $\mathbb{D}^{1}$ and Hongxin Deng ${ }^{1}$

\begin{abstract}
Transcription factors are known to mediate the conversion of somatic cells to induced pluripotent stem cells (iPSCs). Transcription factor TFAP2C plays important roles in the regulation of embryonic development and carcinogenesis; however, the roles of Tfap2c in regulating somatic cell reprogramming are not well understood. Here we demonstrate Tfap2c is induced during the generation of iPSCs from mouse fibroblasts and acts as a facilitator for iPSCs formation. Mechanistically, the c-Myc-dependent apoptosis, which is a roadblock to reprogramming, can be significantly mitigated by Tfap2c overexpression. Meanwhile, Tfap2c can greatly promote mesenchymal-to-epithelial transition (MET) at initiation stage of OSKM-induced reprogramming. Further analysis of gene expression and targets of Tfap2C during reprogramming by RNA-sequencing (RNA-seq) and ChIP-qPCR indicates that TFAP2C can promote epithelial gene expression by binding to their promoters directly. Finally, knockdown of E-cadherin (Cdh1), an important downstream target of TFAP2C and a critical regulator of MET antagonizes Tfap2c-mediated reprogramming. Taken together, we conclude that Tfap2c serves as a strong activator for somatic cell reprogramming through promoting the MET and inhibiting c-Myc-dependent apoptosis.
\end{abstract}

\section{Introduction}

Reprogramming somatic cells to a pluripotent state can be achieved through transient ectopic expression of pluripotency transcription factors (Yamanaka factors), Oct4, Sox2, Klf4, and $c-M y c(\mathrm{OSKM})^{1-3}$. The resulting iPSCs hold significant promise as tools for individualized treatment and regenerative therapy ${ }^{4-6}$. However, the derivation of iPSCs is likely a stochastic event, resulting in very low efficiency $(\sim 0.1 \%$ in humans and $\sim 1.0 \%$ in mice) while being time-consuming ${ }^{1,7}$. Indeed, global transcript and

\footnotetext{
Correspondence: Hongxin Deng (denghongx@scu.edu.cn)

${ }^{1}$ State Key Laboratory of Biotherapy and Cancer Center, West China Hospital,

Sichuan University and Collaborative Innovation Center for Biotherapy, 610041 Chengdu, Sichuan, P.R. China

${ }^{2}$ Department of Obstetrics, Sichuan Provincial Hospital for Women and Children, Chengdu, P.R. China

Full list of author information is available at the end of the article These authors contributed equally: Yuan Wang, Shuang Chen, Qingyuan Jiang Edited by F. Pentimalli
}

protein profiling analysis of intermediates during reprogramming have shown that donor cells undergo a series of phased transitions before reaching the pluripotent state. This process is initiated by the reduction of somatic genes, MET, inhibition of apoptosis and cellular senescence pathways, followed by the upregulation of pluripotency genes, X-chromosome reactivation, telomere elongation and acquirement of the epigenetic characteristics of pluripotent cells ${ }^{8-10}$. In this setup, exogenous factor expression is required for at least 1-2 weeks to establish the endogenous transcriptional network that sustains pluripotency independent of transgene expression. Studies have extensively investigated the molecular mechanisms underlying the formation of iPSCs and sought to identify novel factors that are able to overcome the bottleneck and improve this inherently inefficient process.

Tfap2c (also known as AP2, , Tcfap 2c) belongs to the AP2 transcription factor family, which plays important

\section{(c) The Author(s) 2020}

(c) (i) Open Access This article is licensed under a Creative Commons Attribution 4.0 International License, which permits use, sharing, adaptation, distribution and reproduction c. in any medium or format, as long as you give appropriate credit to the original author(s) and the source, provide a link to the Creative Commons license, and indicate if changes were made. The images or other third party material in this article are included in the article's Creative Commons license, unless indicated otherwise in a credit line to the material. If material is not included in the article's Creative Commons license and your intended use is not permitted by statutory regulation or exceeds the permitted use, you will need to obtain permission directly from the copyright holder. To view a copy of this license, visit http://creativecommons.org/licenses/by/4.0/. 
roles in the regulation of proliferation, cell-cycle control, apoptosis, embryonic development as well as carcinogenesis $^{11,12}$. Mouse Tfap $2 c$ is expressed in both extraembryonic and embryonic tissues and displays multiple functions in trophectoderm formation, neural crest induction and terminal epidermal differentiation ${ }^{13,14}$. Moreover, Tfap $2 c$ is required for the survival of the mouse embryo, Tfap $2 c$ deficient leads to mouse embryonic lethality at approximately embryonic day (E)7.5, which may be attributed to defective placental development ${ }^{15}$. Previous research also revealed the critical roles of Tfap $2 c$ in trophoblast stem cells (TSCs) maintenance and human primordial germ cells (hPGCs) development ${ }^{16-18}$. However, the roles of $T f a p 2 c$ in regulating somatic cell reprogramming and human naïve pluripotency were not reported until recently ${ }^{19-21}$. Transcriptional analysis of poised iPSC intermediates uncovers Tfap $2 c$ is important for the acquisition of pluripotency ${ }^{19}$. More importantly, fibroblasts could be reprogrammed into iPSCs by a novel combination consisted of Gata3, Eomes, Tfap $2 c, c-M y c$, and $E s r r b^{20}$. Despite this, any additional mechanisms accounting for the roles of $T f a p 2 c$ in regulating somatic cell reprogramming are not well understood.

Here we show that Tfap $2 c$ can greatly promote the generation of iPSCs. Mechanistically, Tfap $2 c$ inhibits the $c$-Myc-dependent apoptosis and activates epithelialrelated genes by directly binding to their promoters, resulting in the enhanced MET during reprogramming. Knockdown of Cdh1, an important downstream target of TFAP2C and a critical regulator of MET, antagonizes Tfap $2 c$-mediated reprogramming. Our results provide a mechanistic understanding of Tfap $2 c$-mediated reprogramming, which may facilitate our understanding of the molecular basis of cell identity, pluripotency, and plasticity.

\section{Materials and Methods \\ Mice}

Oct4-GFP transgenic allele-carrying mice (CBA/ $\mathrm{CaJ} \times \mathrm{C} 57 \mathrm{BL} / 6 \mathrm{~J}$ ) were purchased from the Jackson Laboratory. 129S2/SvJaeJ, C57BL/6 J and SCID mice were purchased from Charles River (Beijing, China). Animals (Specific Pathogen Free, SPF) were housed under a 12-h light/dark cycle and provided with enough food and water. Our studies met the requirement of the Care and Use of Laboratory Animals of the National Institutes of Health, and all animal studies were approved by the Ethics Committee of West China Hospital.

\section{Cell culture}

OG2-MEFs were isolated from E13.5 mouse embryos from crossing male Oct4-GFP transgenic allele-carrying mice to 129S2/SvJaeJ female mice. MEFs and HEK293T cells were maintained in DMEM high-glucose media supplemented with 10\% fetal bovine serum (FBS). Mouse ESCs (mESCs) and iPSCs were cultured on feeder layers or feeder free $(0.1 \%$ gelatin; Sigma-Aldrich, MO, USA) with mESC medium. mESC medium consisted of KnockOut $^{\mathrm{TM}}$ DMEM (Gibco, Grand Island, New York, USA) supplemented with 15\% FBS (Gibco, Grand Island, New York, USA), $1 \times$ L-glutamine (Gibco, Grand Island, New York, USA), $1 \times$ NEAA (Gibco, Grand Island, New York, USA), $1 \mathrm{mM}$ sodium pyruvate (Sigma-Aldrich, MO, USA), 1\% penicillin/streptomycin (Gibco, Grand Island, New York, USA), $0.1 \mathrm{mM} \beta$-mercaptoethanol (Sigma-Aldrich, MO, USA), $50 \mathrm{mg} / \mathrm{ml}$ Vitamin C (Sigma-Aldrich, MO, USA), and 1000 units $/ \mathrm{ml}$ leukemia inhibitory factor ((ESG1106, Merck, Darmstadt, Germany). All of the cell lines have been confirmed as mycoplasma contamination free with the kit from Shanghai Yise Medical Technology (PM008).

\section{Retrovirus production and generation of iPSCs}

HEK293T cells were plated at $80-90 \%$ confluency and transfected using polyethylenimine (PEI) transfection method as previous reported ${ }^{22}$. The supernatant of the transfected HEK293T cells were harvested and filtered through a $0.45 \mu \mathrm{m}$ filter (Millipore) after $24 \mathrm{~h}$. Equal volumes of the four supernatants of each OSKM transcription factor with control or Tfap $2 c$ virus were mixed with 1 volume of fresh MEFs medium containing polybrene (Sigma-Aldrich, MO, USA) at a final concentration of $5-8 \mathrm{mg} / \mathrm{ml}$. Two milliliter of infection mixture was used to infect $1.5 \times 10^{4}$ OG2-MEF cells.

For iPSCs generation, $1.5 \times 10^{4}$ OG2-MEFs at passage 2 were plated in a 12 -well plate coated by $0.1 \%$ gelatin and then infected twice with retroviral supernatants. Medium was changed immediately $24 \mathrm{~h}$ after virus transduction and this day is termed as day 0 post-infection. Infected cells were then cultured with mESC medium postinfection and renewed daily. iPSCs colonies appeared about $6-8$ days post infection. Oct4-GFP ${ }^{+}$colonies were counted at day 12 after infection. Flow cytometry was performed to test the Oct4-GFP ${ }^{+}$efficiency. NBT/BCIP (Roche) was used for AP staining according the instructions of the manufacturer.

\section{Immunofluorescence}

Immunofluorescence was performed with standard procedure. The following primary antibodies were used: anti-Nanog (ab80892, abcam), anti-SSEA1 (ab16285, abcam), anti-Ki67 (ab15580, abcam), anti- $\alpha$-fetoprotein (\# MIA1301, Thermo Fisher Scientific, MA, USA), anti- $\alpha$ smooth muscle actin (\#701457, Thermo Fisher Scientific, MA, USA), anti-E-Cadherin (\#14472, Cell Signaling Technology, MA, USA), anti-Epcam (ab71916, abcam), and anti-BIII tubulin (\#MA1-118, Thermo Fisher Scientific, MA, USA). 


\section{shRNAs design and vector construction}

Two pairs of shRNA oligos targeting on Tfap $2 c$ or Cdh1 gene were designed and constructed into PLKO.1 plasmid. The knockdown efficiency was investigated at both mRNA and protein level. The sequences of shRNA oligos are listed in supplementary Table 1.

\section{Quantitative RT-PCR analysis}

Total mRNA was extracted with Trizol Kit (Invitrogen). $0.5 \mu \mathrm{g}$ of total RNA was then reverse transcribed with PrimeScript $^{\mathrm{TM}} \mathrm{RT}$ reagent Kit with gDNA Eraser (Takara, Kusatsu, Japan). Quantitative RT-PCR (qRT-PCR) was performed using TB Green (Takara, Kusatsu, Japan) with a LightCycler $96^{\circledR}$ machine (Roche). The primers used in the qRT-PCR assays are listed in supplementary Table 1.

\section{Embryonic body (EB) formation and teratoma formation}

For embryonic body formation, iPSCs cells were harvested by trypsinization, plated on nonadherent bacterial culture dishes, and incubated in mESC medium without LIF. The colonies were further cultured in suspension for 3 days and then transferred onto gelatin-coated plates. After another continuous culture of 6 days, the cells were collected for later characterization. For teratoma formation $2 \times 10^{6}$ iPSCs were injected subcutaneously into SCID mice. Tumor samples were collected within 4 weeks and processed for immunofluorescence and hematoxylin and eosin (H\&E) staining following standard procedures.

\section{ChIP-qPCR}

ChIP assays were performed using SimpleChIP ${ }^{\circledR}$ Plus Enzymatic Chromatin IP Kit (Cell Signaling Technology, MA, USA) according to the manufacturer's instructions. The primers for ChIP-qPCR are listed in supplementary Table 1.

\section{Cell apoptosis detection}

Reprogramming cells were dissociated with TrypLE ${ }^{\mathrm{TM}}$ Express Enzyme (Gibco) to reduce damage to cells on day 4 post induction. Then cell apoptosis was detected immediately using the Annexin V-APC/PI Apoptosis Detection Kit (4 A Biotech) according to the manufacturer's instructions. In some experimental settings, PAC-1 (Selleck, $1.25 \mu \mathrm{M}$ ) was used to induce apoptosis.

\section{Flow cytometry}

Reprogramming cells were dissociated with TrypLE ${ }^{\mathrm{TM}}$ Express Enzyme (Gibco) at indicated time points. Cells were incubated with PE fluorescently labeled SSEA1 or Epcam monoclonal antibodies (BioLegend, CA, USA) in the dark at $4{ }^{\circ} \mathrm{C}$ for $30 \mathrm{~min}$. After washed twice with PBS, the pellet was resuspended in $400 \mu \mathrm{l}$ PBS and analyzed by the NovoCyte (Acebio).

\section{Immunoblotting}

Immunoblotting was performed with standard procedure. The following antibodies were used in this study: anti-NANOG (\#8822, Cell Signaling Technology, MA, USA), anti-TFAP2C (sc-12762, Santa Cruz Biotechnology, Santa Cruz, CA, USA), anti-GAPDH (\#5174, Cell Signaling Technology, MA, USA), anti-cleaved caspase-3 (\#9664, Cell Signaling Technology, MA, USA), anticleaved PARP1 (ab32064, abcam), anti-CDH1 (\#14472, Cell Signaling Technology, MA, USA), anti- $\beta$-CATENIN (\#8480, Cell Signaling Technology, MA, USA), antiPCNA (\#13110, Cell Signaling Technology, MA, USA), anti-CCND2 (\#3741, Cell Signaling Technology, MA, USA), anti-CCNA2 (\#91500, Cell Signaling Technology, MA, USA), anti-CCNB1 (\#4135, Cell Signaling Technology, MA, USA), anti-CDK1 (\#9116, Cell Signaling Technology, MA, USA), anti-CDK2 (\#2546, Cell Signaling Technology, MA, USA), anti- CCND1 (\#55506, Cell Signaling Technology, MA, USA), and anti-EPCAM (\#93790, Cell Signaling Technology, MA, USA). Immunoblots were visualized on iBright CL1000 Imaging Systems (Thermo Fisher Scientific).

\section{Luciferase activity analysis}

The promoter sequence of $C d h 1$ was cloned into pEZXFR01 (GeneCopoeia) before the Firefly luciferase (Fluc), the Renilla luciferase (Rluc) was used as tracer gene. mESCs were planted in 24-well plates at $2 \times 10^{4}$ per well, and the Tfap2c or control vector $(0.5 \mu \mathrm{g})$ and pEZX-FR01 $(100 \mathrm{ng})$ were cotransfected into the cells with Lipofectamine Stem Transfection Reagent (Invitrogen, STEM00015). At $48 \mathrm{~h}$ after transfection, the luciferase activity was detected according to the instructions for the Luc-Pair Duo-Luciferase Assay Kit 2.0 (GeneCopoeia).

\section{RNA-seq}

Total mRNA was extracted with Trizol Kit (Invitrogen) following the manufacturer's protocol. RNA integrity was evaluated by using the Agilent 2100 Bioanalyzer (Agilent Technologies). The samples with RNA Integrity Number (RIN) $\geq 7$ were subjected to the subsequent testing and analysis. The libraries were constructed using TruSeq Stranded mRNA LTSample Prep Kit (Illumina) according to the manufacturer's instructions. Then these samples were sequenced on the Illumina sequencing platform (Illumina HiSeq $\times$ Ten) and 150 bp paired-end reads were generated. All of these procedures were performed by Chengdu Basebiotech Co., Ltd. And the original data of the transcription array were uploaded into SRA (SRP234686).

\section{Statistical analysis}

For statistical analysis, mean values with \pm SD . were presented in most graphs that were derived from at least 
three repeats of biological experiments $(N=3)$. Differences between two datasets were calculated using Unpaired two-tailed Student's $t$-test, one-way ANOVA with Dunnett's test, and two-way AVOVA with Sidak's multiple comparisons test with $P<0.05$ considered statistically significant. Statistical analysis was performed in Prism 8 (Graphpad Inc.). Sample sizes and specific tests are indicated in the figure legends. ${ }^{*} P<0.05,{ }^{* *} P<0.01$,

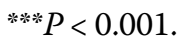

\section{Results}

\section{Tfap2c is upregulated during the induction of iPSCs}

To investigate the roles of $T f a p 2 c$ in regulating reprogramming, we first examined Tfap $2 c$ expression levels in mouse embryonic fibroblasts (MEFs), mouse embryonic stem cells (ESCs), and iPSCs by reanalyzing a published GEO DataSets (Accession: GSE66613). Tfap2c was significantly higher expressed in ESCs and iPSCs compared with MEFs (Supplementary Fig. S1a). The results can be further confirmed by western blot and quantitative PCR with reverse transcription (qRT-PCR) with Nanog as a positive control (Supplementary Fig. S1b, c). We also noticed a gradual increase in Tfap $2 c$ expression during the reprogramming of MEFs with OSKM factors (Supplementary Fig. S1d). By using MEFs derived from transgenic mice carrying Oct4-GFP/Rosa26 (OG2-MEF), we could isolate SSEA1 positive (SSEA1 ${ }^{+}$) and Oct4-GFPpositive $\left(O c t 4-\mathrm{GFP}^{+}\right)$populations, which are known intermediates poised to reprogram ${ }^{19,23}$. We found Tfap $2 c$ was higher expressed in SSEA1 ${ }^{+}$and Oct4-GFP $^{+}$cells compared with SSEA1-negative (SSEA1 ${ }^{-}$) and Oct4-GFPnegative (Oct4-GFP $\left.{ }^{-}\right)$cells, respectively (Supplementary Fig. S1e, f). Previous research has demonstrated the Tfap $2 c$ promoter is co-occupied by OCT4, SOX2, and KLF4, indicating that cooperative binding may be responsible for the activation of Tfap $2 c^{19,24}$. To test this hypothesis, we examined the effect of withdrawal of individual factor from OSKM on the activation of Tfap2c. Indeed, withdrawal of any factors from OSKM impaired the expression of Tfap2c (Supplementary Fig. S1g). These results indicate that during the induction of iPSCs, Tfap $2 c$ is upregulated by OSKM cooperatively, and is preferentially activated in intermediates poised to reprogram, indicating a potential role of Tfap $2 c$ in regulating reprogramming.

\section{Tfap2c is a facilitator of somatic cell reprogramming}

To investigate $T f a p 2 c$ function in reprogramming, we transduced Tfap $2 c$ along with OSKM into MEFs and assessed the effect of Tfap $2 c$ overexpression in MEFs reprogramming (Supplementary Fig. S2a). Tfap $2 c$ overexpression promoted the formation of alkaline phosphatase (AP)-positive colonies (an early marker of reprogramming) and resulted in a significant increase in the fraction of SSEA $1^{+}$and $O c t 4-\mathrm{GFP}^{+}$(a late marker of reprogramming) intermediates compared with controls (Fig. 1a-d). Furthermore, we observed that Tfap2c overexpression significantly promoted the appearance of Oct4-GFP ${ }^{+}$colonies (Fig. 1e, f). The OSKM+Tfap $2 c$ iPSCs (OSKMT-iPSCs) exhibited typical ESCs morphology, with a compact appearance and a well-defined border (Supplementary Fig. S3a). Immunofluorescence staining and qRT-PCR indicated that OSKMT-iPSCs, but not MEFs, exhibited expression of pluripotent genes at both mRNA and protein levels comparable with that of ESCs (Supplementary Fig. S3b-d). We then conducted in vitro and in vivo differentiation assays to investigate the differentiation potential of OSKMTiPSCs. After 9 days of embryoid body (EB)-mediated in vitro differentiation (Supplementary Fig. S3e), the differentiated cells were positive for markers of three germ layers, including $\alpha$-fetoprotein (endoderm marker), $\alpha$-smooth muscle actin (mesoderm marker) and $\beta I I I$ tubulin (ectoderm marker). Teratomas also developed after subcutaneous injection of OSKMT-iPSCs into SCID mice, resulting tissues with histological structures characteristic of the three germ layers, which were further characterized by the expression of specific markers (Supplementary Fig. S3f). Thus, the OSKMTiPSCs are functional pluripotent stem cells, as they were able to differentiate into all three germ layers in vivo and in vitro. Next, we constructed two short hairpin RNA (shRNA) vectors that showed robust downregulation of both the mRNA and protein of Tfap $2 c$ (Supplementary Fig. S2b). Consistent with the results of overexpression experiments, knockdown Tfap $2 c$ prevented the formation of iPSCs (Fig. 1g-l). Given the robust effects of $T f a p 2 c$ in reprogramming, we speculated that Tfap $2 c$ may be able to substitute any of the Yamanaka factors. We found that, although Tfap $2 c$ could not replace Oct4, Sox2, and Klf4, it indeed could 'replace' $c-M y c$ to yield comparable $O c t 4-\mathrm{GFP}^{+}$colonies (Supplementary Fig. S4a). In addition, OSKT also generated more Oct4-GFP ${ }^{+}$colonies compared with OSK (Supplementary Fig. S4a-e). Taken together, our data indicated that Tfap $2 c$ is a facilitator of both OSK- and OSKM-induced somatic cell reprogramming.

\section{RNA-seq analysis of the effects of Tfap2c on somatic cell reprogramming}

To further confirm the effects of Tfap $2 c$ on reprogramming, we performed RNA-seq on day 4 and day 8 post induction. Tfap $2 c$ overexpression resulted in extensive transcriptional changes, and in line with the transcription activator activity of TFAP2C ${ }^{25,26}$, most genes were upregulated (Fig. 2a, b). By comparing Tfap $2 c$-affected genes (2-fold change in expression, $P<0.05$ ) with a list of 'signature' reprogramming genes that are dynamically 


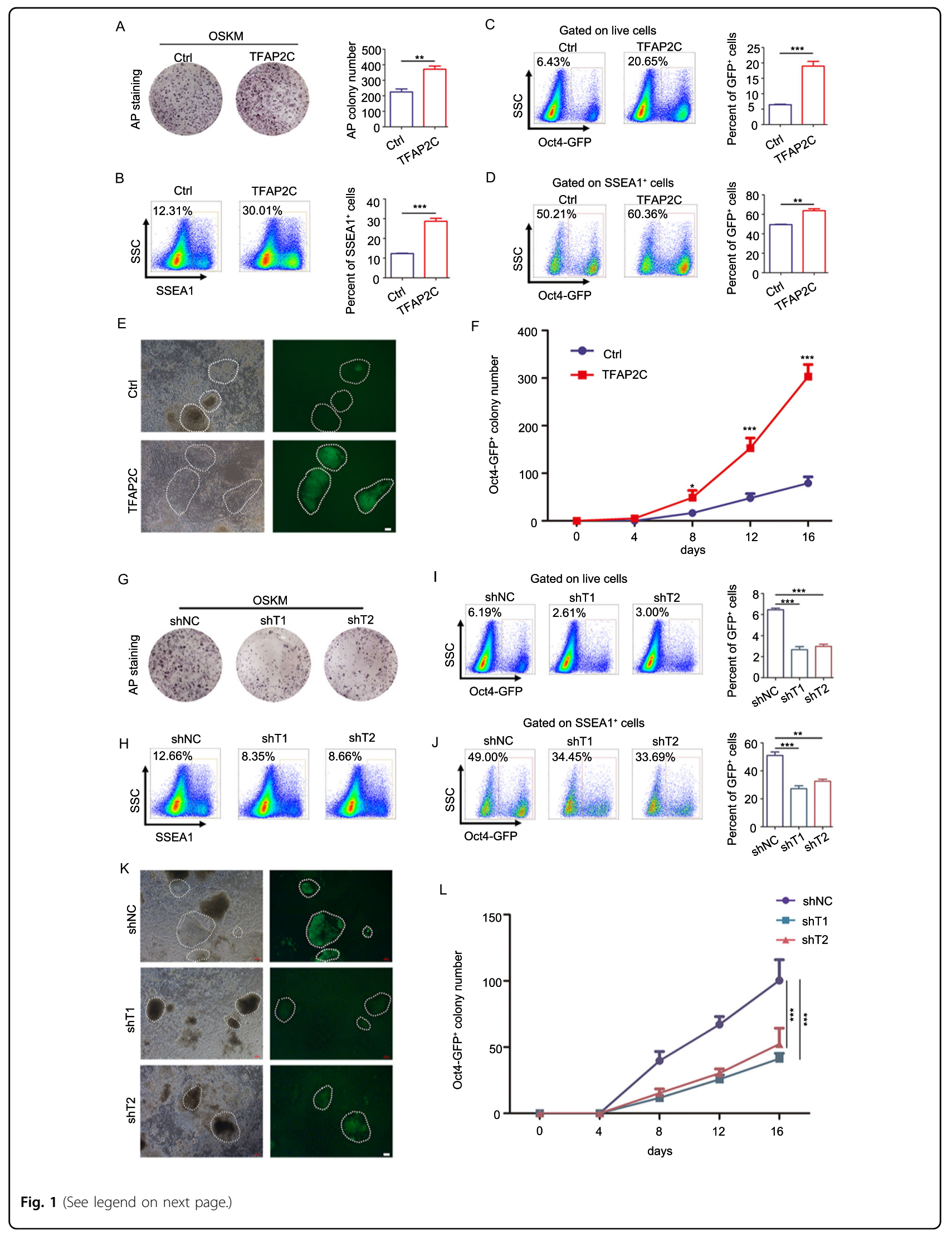


(see figure on previous page)

Fig. 1 Tfap2c is a facilitator of somatic cell reprogramming. a AP-staining wells of a representative reprogramming experiment transduced with OSKM or OSKMT (day 9). b Flow-cytometer analysis of the SSEA $1^{+}$cells in reprogramming cells transduced with OSKM or OSKMT (day 9). c Flowcytometer analysis of the Oct4-GFP+ cells in living cell population transduced with OSKM or OSKMT (day 12). d Flow-cytometer analysis of the Oct4$\mathrm{GFP}^{+}$cells in SSEA $1^{+}$cell population transduced with OSKM or OSKMT (day 12). e Image of Oct4-GFP ${ }^{+}$colonies generated from MEFs transduced with OSKM or OSKMT in bright and fluorescent views (day 9). Scale bar, $100 \mu \mathrm{mm}$. $\mathbf{f}$ Number of Oct4-GFP ${ }^{+}$colonies of MEFs reprogrammed with OSKM and OSKMT at different time points. $\mathbf{g}$ AP-staining wells of a representative reprogramming experiment transduced with OSKM and either negative control shRNA or two shRNAs targeting Tfap2C (shT1 and shT2) on day 9. $\mathbf{h}$ Flow-cytometer analysis of the SSEA ${ }^{+}$cells in reprogramming cells in different groups (day 12). i Flow-cytometer analysis of the Oct4-GFP+ cells in living cell population in different groups (day 12). $\mathbf{j}$ Flow-cytometer analysis of the Oct4-GFP ${ }^{+}$cells in SSEA1 ${ }^{+}$cell population in different groups (day 12). $\mathbf{k}$ Bright and fluorescent views of Oct4-GFP ${ }^{+}$colonies in different groups (day 12). Scale bar, $100 \mu \mathrm{m}$. I Number of Oct4-GFP ${ }^{+}$colonies in different groups at different time points. Significance in panels a, b, c, and $\mathbf{d}$ was determined by Unpaired two-tailed Student's $t$-test. Significance in panels $\mathbf{f}$ and $\mathbf{I}$ was determined by two-way AVOVA with Sidak's multiple comparisons test. Significance in panels $\mathbf{i}$ and $\mathbf{j}$ was determined by one-way ANOVA with Dunnett's test. Significance summary: ${ }^{*} P \leq 0.05$; ${ }^{* *} P \leq 0.01 ;{ }^{* * *} P \leq 0.001$. All data are presented as mean \pm S.D., $N=3$.

regulated in reprogramming ${ }^{27,28}$, we found many genes (Cdh1, Cldn3, Nanog, and Lin28a) that are beneficial to reprogramming were upregulated, and genes which inhibit reprogramming were downregulated (Mex2, Jag2, and Col6a2) (Fig. 2c, d, Supplementary Table 2). Gene ontology (GO) analysis revealed that genes upregulated by Tfap $2 c$ are exceptionally enriched for cell-cell adhesion processes at day 4 (Fig. 2e). Other terms include those related to cell morphology, development, autophagy as well as stem cell population maintenance (Fig. 2e, f), all of which are critical events in reprogramming ${ }^{29-32}$. In addition, Kyoto Encyclopedia of Genes and Genomes (KEGG) analysis showed signal pathways that are essential for reprogramming, including focal adhesion, autophagy, and glycolysis were widely affected by Tfap $2 c$ overexpression (Supplementary Fig. S5). In conclusion, these results demonstrate that $T f a p 2 c$ can promote reprogramming at the transcriptome level.

\section{Tfap2c exerts its effect at the beginning of the reprogramming process}

As somatic cell reprogramming can be divided into phases with distinct molecular and phenotypic features, and factors that regulate iPSCs generation can function at different phases during reprogramming ${ }^{32}$, we next sought to determine the time window during which Tfap $2 c$ can promote somatic cell reprogramming. To this end, we developed an inducible gain-of-function approach and showed that Tfap $2 c$ can be induced in Tfap $2 c^{\text {TetOn }}$ MEFs by doxycycline (Dox) at both mRNA and protein level (Fig. $3 \mathrm{a}-\mathrm{c}$ ). Then, we induced Tfap $2 c$ expression for different durations during the somatic cell reprogramming process and examined the percentage of Oct4-GFP ${ }^{+}$cells and the number of Oct4-GFP ${ }^{+}$colonies at the end of reprogramming (day 12). We found that ectopic expression of Tfap $2 c$ in the first 2 days significantly enhanced the reprogramming efficiency (Fig. $3 \mathrm{~d}-\mathrm{f}$ ). The efficiency could be slightly increased and reached a peak if Tfap $2 c$ is activated in the first 4 days, further overexpression of
Tfap $2 c$ could not affect reprogramming significantly (Fig. 3d-f). As a complement, we also induced Tfap $2 c$ expression beginning at different days after the initiation of reprogramming and found that Tfap $2 c$ overexpression after day 4 showed no obvious effect on reprogramming (Fig. 3d-f). In conclusion, these results suggested that Tfap $2 c$ plays an important role in promoting iPSCs generation at the initiation phase (0-4 day) of reprogramming.

\section{Tfap2c inhibits c-Myc-dependent apoptosis}

Given that Tfap $2 c$ exerts its effect at the beginning of the reprogramming, a phase which is characterized by the reduction of somatic genes, MET, inhibition of apoptosis and cellular senescence pathways $s^{5,33-35}$, we speculated Tfap $2 c$ may promote reprogramming by regulating these relevant events. Intriguingly, we observed a significant increased cell number upon Tfap $2 c$ overexpression (Fig. 4a). We then investigated the effect of Tfap $2 c$ on cell proliferation, cycle, and apoptosis. Although Tfap $2 c$ had limited effect on cell proliferation and cell cycle (Fig. 4b, Supplementary Fig. S6a), it inhibited apoptosis significantly, which was evaluated by reduction of Annexin $\mathrm{V}^{+}$cells and the decreased expression of cleaved caspase-3 (CC3) and cleaved PARP1 (CPARP1) (Fig. 4c-f). Indeed, RNA-seq analysis also indicated that genes involving in positive regulation of apoptosis were downregulated, while that involving in negative regulation of apoptosis were upregulated by Tfap $2 c$ (Supplementary Table 3). We further demonstrated that knockdown of Tfap 2c increased the expression of CC3 and CPARP1 in OSKMinduced reprogramming (Supplementary Fig. S6b). Interestingly, the apoptosis level was much lower in OSK-induced reprogramming compared with that in OSKM-induced reprogramming and Tfap $2 c$ did not change apoptosis level in OSK-induced reprogramming (Fig. 4c-f). These results are consistent with prior findings that ectopic $c-M y c$ expression sensitizes cells to 


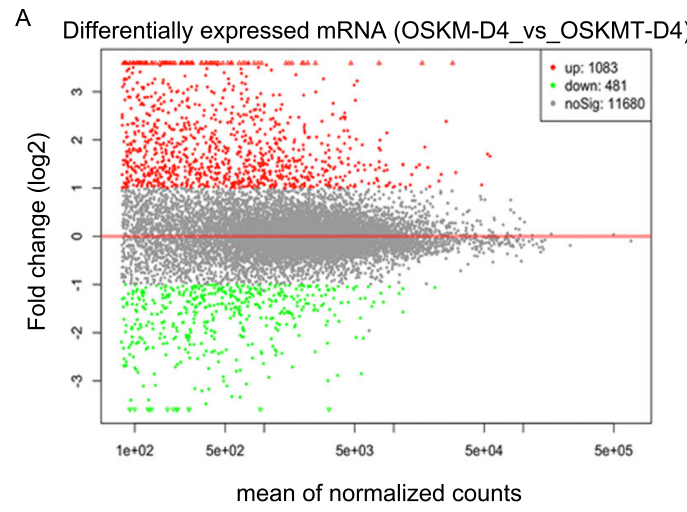

C

\begin{tabular}{|ll|}
\hline Meox2 & Sall1 \\
Tead2 & Crb3 \\
Col6a2 & Cldn3 \\
Cdh1 & Cldn4 \\
Epcam & Irf6 \\
& ... \\
\hline
\end{tabular}
genes from RNAi or CRISPRa screening

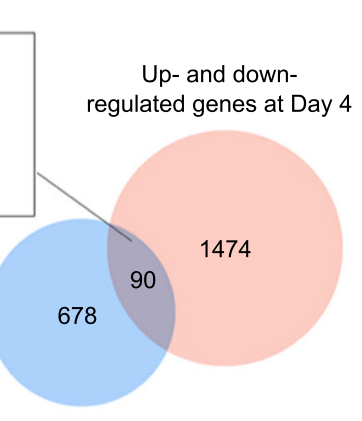

B Differentially expressed mRNA (OSKM-D8_vs_OSKMT-D8)

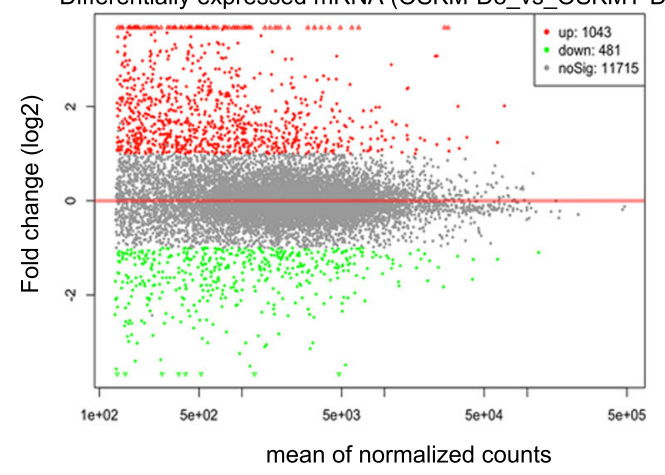

D

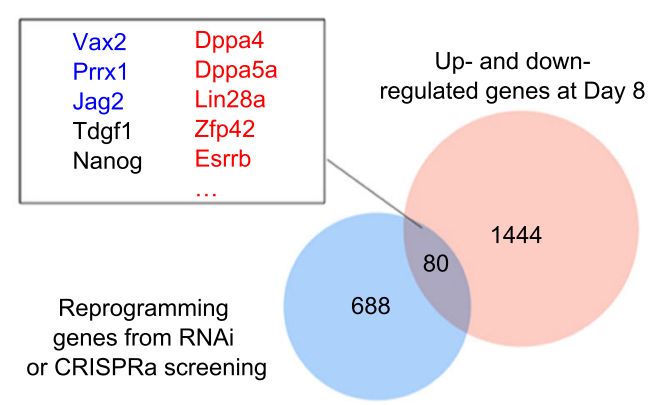

$\mathrm{E}$

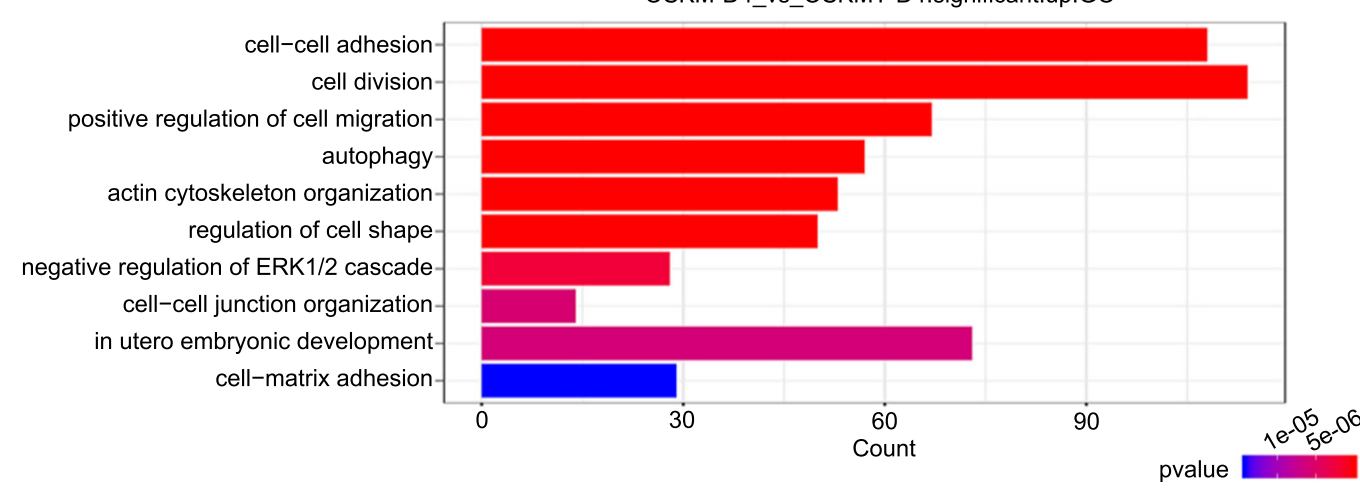

$\mathrm{F}$

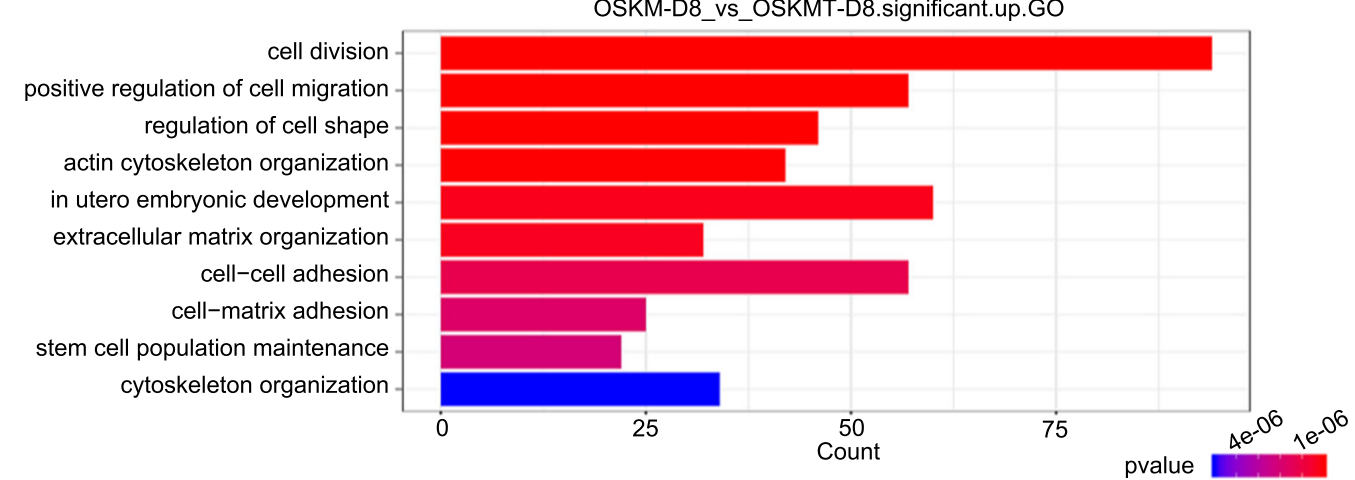

Fig. 2 RNA-seq analysis of the effects of Tfap2c on somatic reprogramming. a Number of differentially regulated genes after Tfap2C overexpression compared with control on day 4 . b Number of differentially regulated genes after Tfap2c overexpression compared with control on day 8. c Venn diagram showing the common genes between Tfap2c-affected genes (day 4) and the previously identified reprogramming genes. Genes that impair (or facilitate) reprogramming and downregulated (or upregulated) simultaneously in our RNA-seq dataset are highlighted in blue (or red). $\mathbf{d}$ Venn diagram showing the common genes between Tfap2c-affected genes (day 8) and the previously identified reprogramming genes. e Gene ontology analysis for genes upregulated by Tfap2c on day 4. f Gene ontology analysis for genes upregulated by Tfap2c on day 8 . 


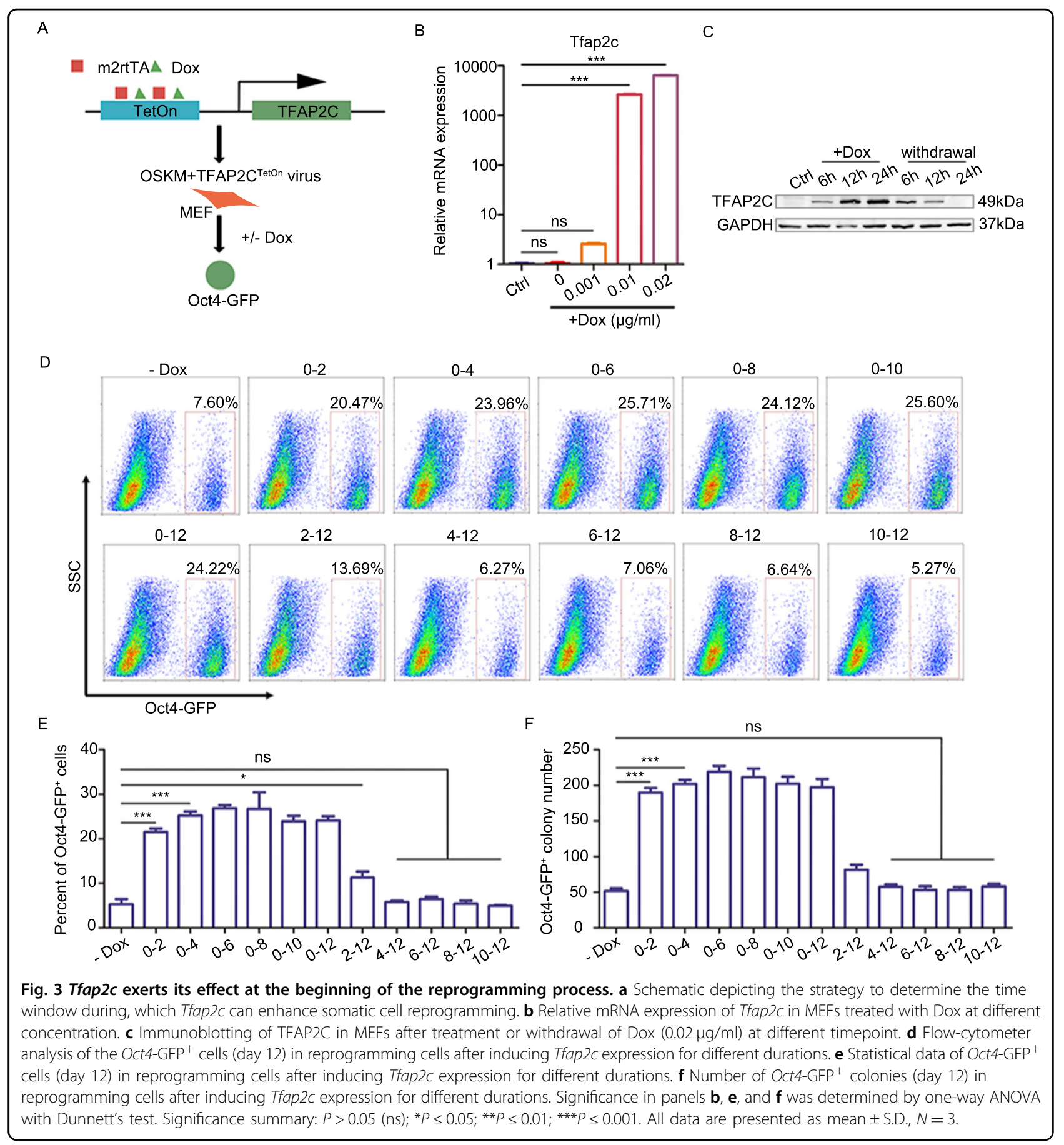

apoptosis $^{36}$. To investigate whether the ability of Tfap $2 c$ to inhibit apoptosis requires the help of OSK, we detected the antiapoptotic effects of Tfap $2 c$ in the absence of OSK. Our results indicated that $c-M y c$ alone could trigger apoptosis in MEFs and Tfap $2 c$ could inhibit apoptosis in the absence of OSK (Supplementary Fig. S6c). We further induced apoptosis by treating cells with low dose $(1.25 \mu \mathrm{M})$ of PAC-1, a small molecule that could induce apoptosis by enhancing the enzymatic activity of procaspase-3. We found PAC-1 could promote the expression of CC3 and CPARP1 and antagonize the antiapoptotic effects of Tfap $2 c$ partly (Fig. $4 \mathrm{~g}, \mathrm{~h}$ ), which led to a lower reprogramming efficiency. The above data indicated Tfap 2c can inhibit $c-M y c$-dependent apoptosis in OSKM-induced reprogramming. 


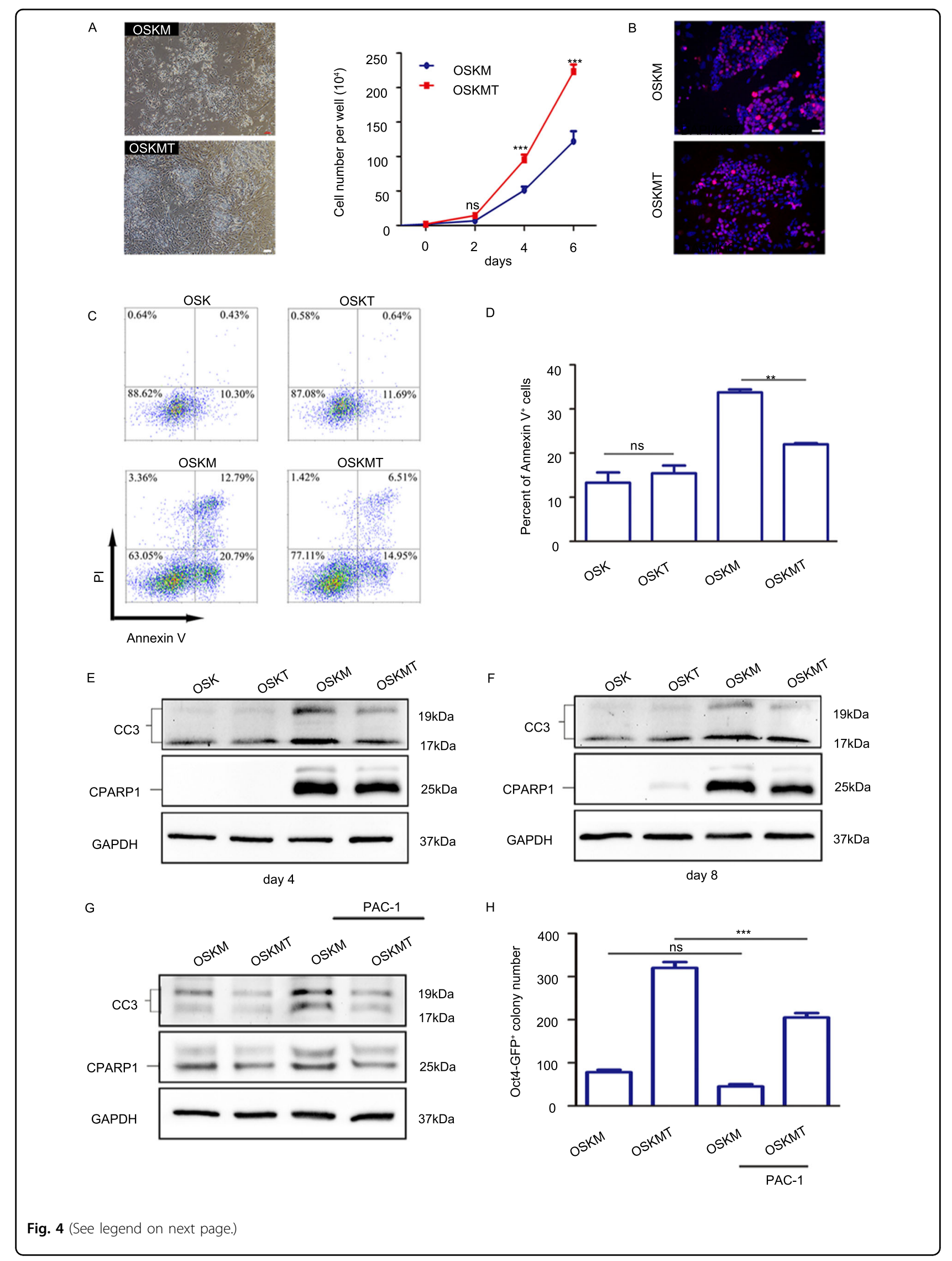


(see figure on previous page)

Fig. 4 Tfap2c inhibits c-Myc-dependent apoptosis. a Microscopy of reprogramming cells at post-infection day 4, and the number of OSKM and OSKMT reprogramming cells at different time points was recorded. Scale bar, $100 \mu \mathrm{m}$. b Immunostaining of Ki67 in OSKM and OSKMT groups at day 4. Scale bar, $50 \mu \mathrm{m}$. c Flow-cytometer analysis of cell apoptosis after infected with OSK, OSKT, OSKM, and OSKMT on day 4. d The percent of Annexin $\mathrm{V}^{+}$cells in OSK, OSKT, OSKM, and OSKMT group on day 4. e Immunoblotting of Cleaved caspase-3 (CC3) and Cleaved PARP1 (CPARP1) in MEFs after infected with OSK, OSKT, OSKM, and OSKMT on day 4. f Immunoblotting of CC3 and CPARP1 in MEFs after infected with OSK, OSKT, OSKM, and OSKMT on day 8. $\mathbf{g}$ Immunoblotting of CC3 and CPARP1 in MEFs after infected with OSKM and OSKMT (day 4) with or without PAC-1 (1.25 $\mu M$ ) treatment. $\mathbf{h}$ Number of Oct4-GFP+ colonies (day 12) in reprogramming cells treated with or without PAC-1. Significance in panels $\mathbf{d}$ was determined by one-way ANOVA with Dunnett's test. Significance in panels $\mathbf{a}$ and $\mathbf{h}$ was determined by two-way AVOVA with Sidak's multiple comparisons test. Significance summary: $P>0.05$ (ns); ${ }^{*} P \leq 0.05 ;{ }^{* * *} P \leq 0.001$. All data are presented as mean \pm S.D., $N=3$.

\section{Tfap2c promotes MET at the initiation stage of reprogramming}

The fact that Tfap $2 c$ promotes both OSK- and OSKMinduced reprogramming, while the apoptosis level is unaffected by $T f a p 2 c$ in OSK-induced reprogramming prompt us to find other mechanisms that Tfap $2 c$ may regulate reprogramming. Another essential hallmark in the initiation phase of reprogramming is $\mathrm{MET}^{9,37}$. Indeed, GO and KEGG analysis revealed that genes upregulated by $T f a p 2 c$ are exceptionally enriched for cell-cell adhesion and focal adhesion processes, both of which are key events involved in MET (Fig. 2e, f, Supplementary Fig. S5). In addition, Tfap $2 c$ promoted the conversion of fibroblast-like cells into epithelial-like cell at as early as day 2 (Supplementary Fig. S7). To further explore the role of $T f a p 2 c$ on MET, we checked the expression levels of the key epithelial and mesenchymal markers in our RNAseq dataset. Tfap $2 c$ overexpression leads to significant upregulation of epithelial genes, including Cdh1, Epcam, Cldn3, and Krt8. Consistent with the activation of epithelial genes, the expression level of mesenchymal regulators, such as Twist1, Twist2, Zeb2, Tgfb2, and Zeb1, were markedly downregulated after Tfap $2 c$ overexpression (Fig. 5a). To confirm this, we examined the mRNA expression level of epithelial- and mesenchymalassociated genes during reprogramming by qRT-PCR (Fig. 5b, c). Flow cytometry, western blot, and immunofluorescence analysis further validated the increased expression of epithelial regulators (Fig. 5e, f, Supplementary Fig. S8a, b). In addition, epithelial genes were downregulated after $T f a p 2 c$ knockdown in reprogramming (Supplementary Fig. S8c). More importantly, Tfap $2 c$ also facilitated MET in OSK-induced reprogramming (Supplementary Fig. S9). Tfap $2 c$ alone could also promote MET moderately in the absence of OSKM; however, the degree was lower than that in reprogramming (Supplementary Fig. S8d). Pluripotent markers including Nanog, Lin28a, and Dppa5a were generally unaffected until day 8 (Fig. 5d, f). Given that Tfap $2 c$ exerts its effect from the beginning of reprogramming, we speculated that Tfap $2 c$ directly contributes to the earlier MET program, whereas its effect on the later pluripotent program is the result of an amplified cascade. Collectively, the data presented above indicated that $T f a p 2 c$ facilitates MET, which may in turn activate downstream genes including pluripotent genes.

\section{Tfap2c activates MET regulators directly}

To figure out how $T f a p 2 c$ regulates MET, we performed chromatin immunoprecipitation (ChIP)-qPCR given that canonical TFAP2C-binding motif GCCNNNGGC exist in the promoter regions of many MET genes (Fig. 6a). Our results demonstrated that the genomic loci of these MET genes were occupied by TFAP2C (Fig. 6b), indicating TFAP2C may activate MET by binding the promoter directly. Among many of the TFAP2C targets and MET regulators, we selected $C d h 1$ as an important candidate, which may meditate $T f a p 2 c$-induced reprogramming for its multifunction roles in cell-cell adhesion and maintenance of pluripotency ${ }^{38-42}$. We then mutated the canonical motif of TFAP2C in the $C d h 1$ promoter and showed that it blocked the activation effects of TFAP2C in a reporter assay (Fig. 6c, d). Next, we constructed two shRNA vectors that showed robust downregulation of both the mRNA and protein of Cdh1 (Fig. 6e, f, Supplementary Fig. S10a). Indeed, knockdown of $C d h 1$ impaired the expression of epithelial and pluripotent genes (Fig. 6e) and led to a blocked MET (Supplementary Fig. S10b) and pluripotent program. More importantly, the number of $\mathrm{AP}^{+}$and Oct4-GFP ${ }^{+}$colonies were decreased remarkably upon inhibition of Cdh1 (Fig. 6g, Supplementary Fig. S10c, d). Collectively, our results demonstrated that Tfap $2 c$ promotes reprogramming by regulating MET directly.

\section{Discussion}

In this study, we report that the transcription factor $T f a p 2 c$ acts as a facilitator to somatic cell reprogramming. Mechanistically, Tfap $2 c$ inhibits the $c-M y c$-dependent cell apoptosis and activates epithelial-related genes by binding to the promoter directly, resulting in the enhanced MET and reprogramming efficiency (Fig. 6h).

Since the first report of iPSCs, the molecular mechanisms of reprogramming have been extensively investigated. A better mechanistic understanding of reprogramming is essential to iPSCs biology and improvement of the 


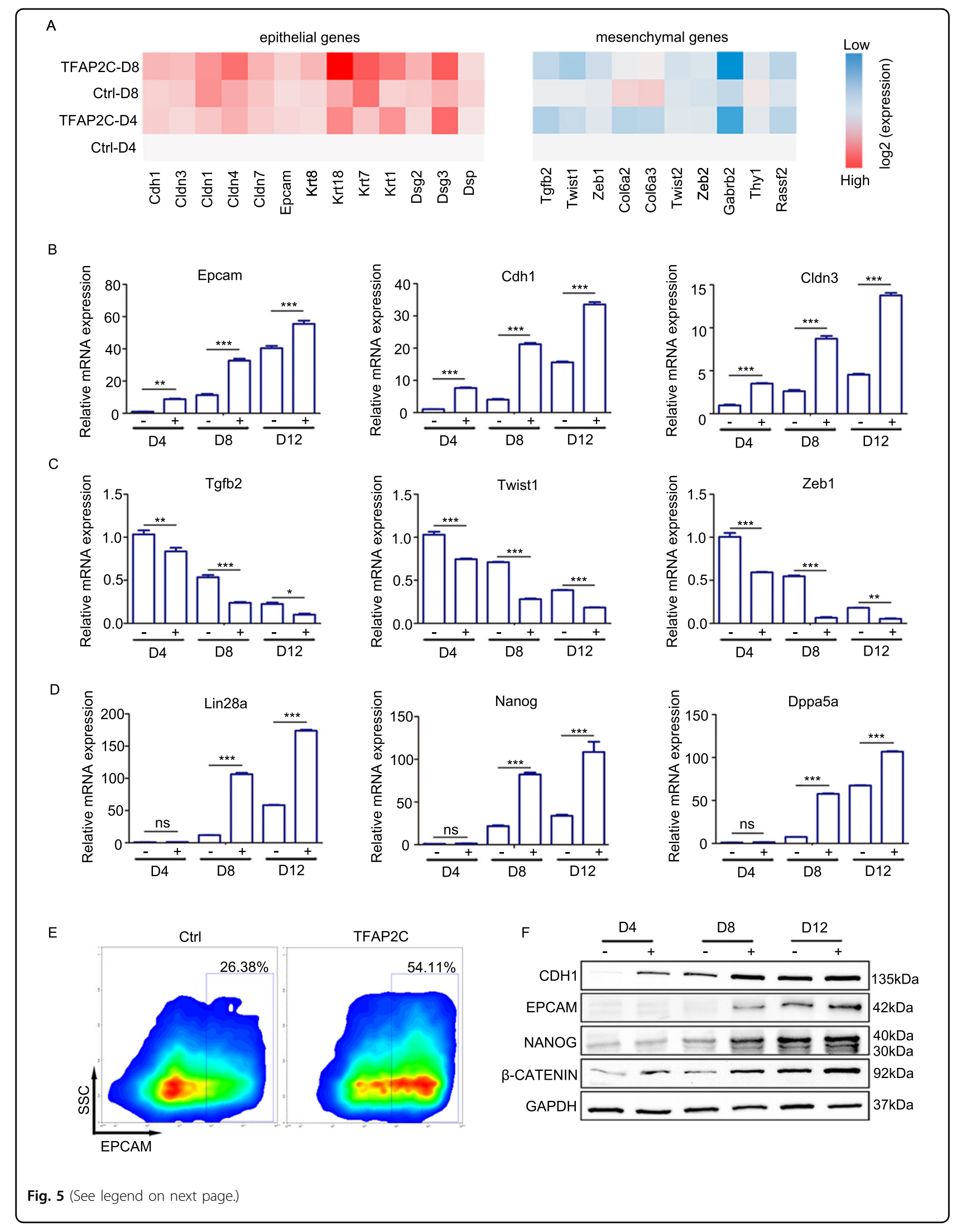


(see figure on previous page)

Fig. 5 Tfap2c promotes MET at the initiation stage of reprogramming. a Heatmap of the epithelial and mesenchymal genes in reprogramming cells transduced with OSKM or OSKMT at day 4 and day 8 post induction. b qRT-PCR analysis of epithelial genes expression in reprogramming cells transduced with OSKM (-) or OSKMT (+) at indicated time points. c qRT-PCR analysis of mesenchymal genes expression in reprogramming cells transduced with OSKM or OSKMT at indicated time points. $\mathbf{d}$ QRT-PCR analysis of pluripotential genes expression in reprogramming cells transduced with OSKM or OSKMT at indicated time points. e Flow-cytometer analysis of the EPCAM ${ }^{+}$cells in reprogramming cells transduced with OSKM or OSKMT on day 9. $\mathbf{f}$ Immunoblotting of CDH1, EPCAM, $\beta$-CATENIN, and NANOG in reprogramming cells transduced with OSKM or OSKMT at indicated time points. Significance in panels $\mathbf{b}, \mathbf{c}$, and $\mathbf{d}$ was determined by two-way AVOVA with Sidak's multiple comparisons test. Significance summary: $P>0.05$ (ns); ${ }^{*} P \leq 0.05 ;{ }^{* *} P \leq 0.01 ;{ }^{* *} P \leq 0.001$. All data are presented as mean \pm S.D., $N=3$.

efficiency and quality of iPSCs for therapeutic use. Typically, introduction of factors that are highly expressed in ESCs, such as Lin28a and Nanog, while inhibition of genes that show low expression in ESCs, such as $c$-Jun and $p 53$, augments reprogramming ${ }^{43-45}$. In our study, Tfap $2 c$ was significantly higher expressed in ESCs and iPSCs compared with MEFs and was preferentially activated in intermediates poised to reprogram, indicating a potential role of Tfap $2 c$ in promoting somatic cell reprogramming. In addition, we found OSKT could generate comparable Oct4-GFP ${ }^{+}$colonies compared with OSKM, which may avoid undesirable genetic modification in reprogramming caused by oncogenes $c-M y c^{46}$. Collectively, our results highlight the important potential of Tfap $2 c$ regarding the efficiency and safety in reprogramming, which are key issues for the further application of iPSCs.

Mechanistic analyses have demonstrated that the first step of reprogramming is "initiation", and it is characterized by loss of somatic cell gene expression, changes of metabolism and cytoskeleton organization, increase in cellular proliferation, inhibition of apoptosis and initiation of $\mathrm{MET}^{47-49}$. Given that Tfap2c exerts its effects at the initiation phase of the reprogramming, we speculated Tfap $2 c$ may promote reprogramming by regulating relevant events involving in this phase. Cell apoptosis has been identified as a barrier to reprogramming ${ }^{35,50,51}$, one of the well-characterized mechanisms is the activation of p53 pathway and the accompanying cell senescence and apoptosis ${ }^{33,50}$. In addition, although $c-M y c$ enhances the overall reprogramming efficiency, $c-M y c$ can also trigger cell apoptosis via $p 53$-dependent and -independent way, which may limit reprogramming ${ }^{35,50}$. This seems counterintuitive since exogenous $c-M y c$ has traditionally been considered as a facilitator for reprogramming. However, recent studies had revealed dual role of $c-M y c$ in reprogramming, and indicated $c-M y c$ can negatively influences reprogramming by inducing cell apoptosis and creating an epigenetic barrier together with NCoR/SMRT corepressors $^{52}$. In our present study, we found Tfap $2 c$ can attenuated the 'side effect' of $c-M y c$ in reprogramming by inhibiting $c-M y c$-induced apoptosis, in agreement with previous results that Tfap $2 c$ decreased apoptosis in an activated $\mathrm{Neu}$ model of mammary carcinogenesis ${ }^{53}$. Although the precise mechanism is remained to further investigated, the fact that AP2 transcription factor family function as a negative regulator of $c-M y c$ by impairing DNA binding of $c-M y c$ at specific sites prompts us to speculate Tfap $2 c$ may inhibit $c-M y c$-dependent apoptosis in similar way ${ }^{54}$.

Accumulating evidence indicates that MET is indispensable during early somatic cell reprogramming in mouse embryonic fibroblasts and human fibroblasts, and that blocking this step can immensely impair reprogramming efficiency ${ }^{39}$. During MET, mesenchymal cells progressively undergo the establishment of apicobasal polarity, which accompanied by formation of tight junctions and the reorganization of cytoskeletal structures ${ }^{9}$. It has been reported that Tfap $2 c$ played important role in the identity of a wide variety of ectodermal and endodermal-derived epithelia, including mammary, colon, and skin epidermis ${ }^{55-58}$. For example, loss of Tfap $2 c$ in luminal breast cancer cells induced a luminal to basal cell transition and was associated with the development of a mesenchymal expression pattern ${ }^{42}$. In our present study, we found genes upregulated by $T f a p 2 c$ are exceptionally enriched for cell-cell adhesion processes, cell morphology, cytoskeleton organization as well as cell migration. In addition, Tfap $2 c$ overexpression lead to significant upregulation of epithelial genes by binding to the promoter directly. Given the potential of TFAP2C in regulating $C d h 1$ and the indispensable role of Cdh1 in MET, somatic cell reprogramming and maintenance of pluripotency ${ }^{38,39,41,42}$, we selected $C d h 1$ as an important candidate, which may meditate Tfap $2 c$ induced reprogramming. Our data demonstrated that downregulation of the expression of Cdh1 significantly antagonized the effect of Tfap $2 c$-meditated reprogramming, further emphasizing the critical role of MET in reprogramming.

Tfap $2 c$ is known for its central role in trophoblast development and in the conversion of mouse fibroblasts into functional-induced trophoblast stem-like cells $(\mathrm{iTSCs})^{14,17}$. Previous and our present studies also demonstrate the importance of Tfap $2 c$ in somatic cell reprogramming ${ }^{19,23}$. More importantly, a combination of five transcription factors including Tfap2c (Gata3, Tfap2c, Eomes, c-Myc, and Esrrb) can reprogram mouse fibroblasts into both iPSCs and iTSCs ${ }^{20}$. These results agree with the 
A

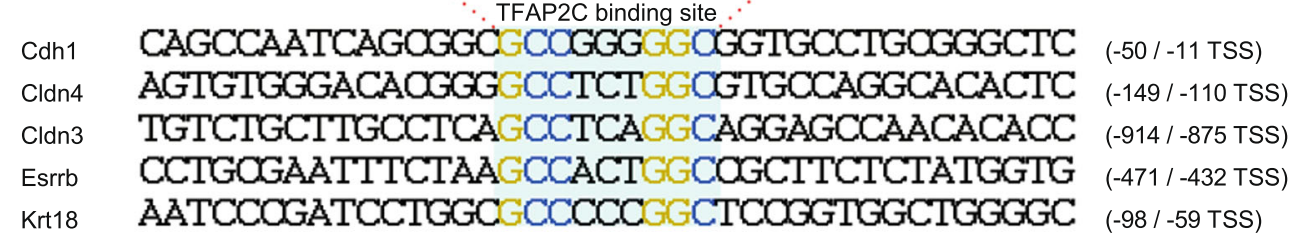

B
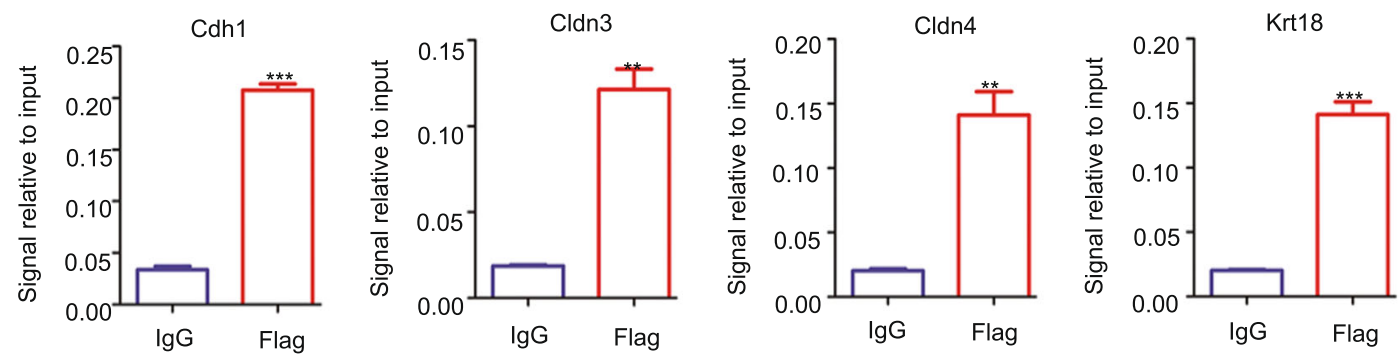

C -50 TSS Cdh1 promoter

CAGCCAATCAGCGGC GCCGGGGGC GGTGCCTGCGGGCTC
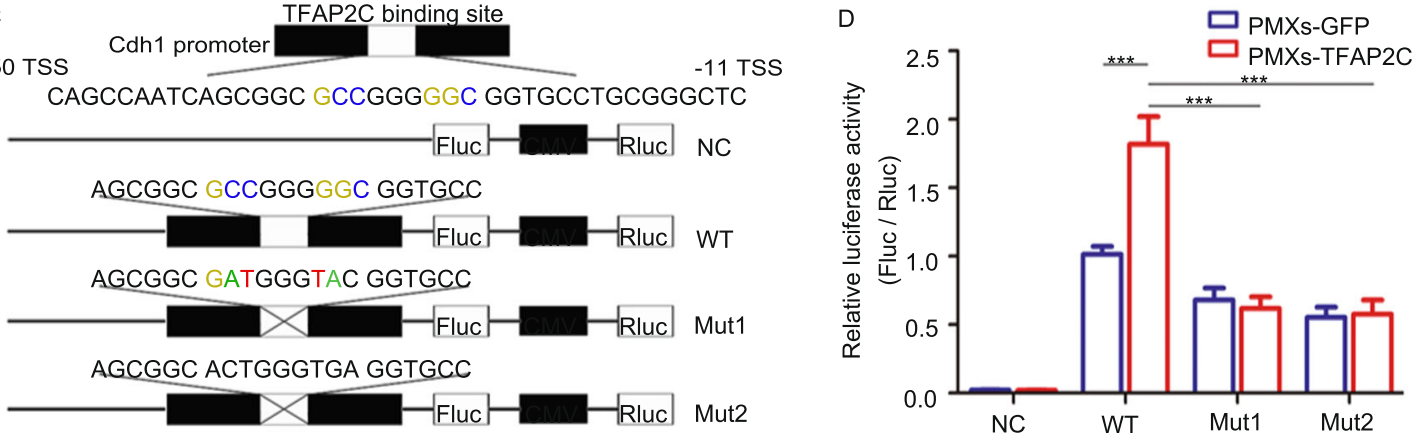

$\mathrm{E}$

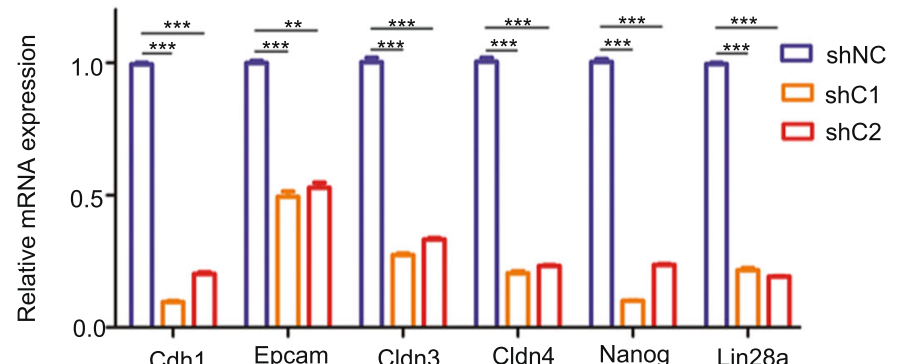

$\mathrm{F}$

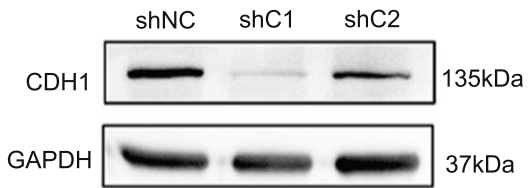

G

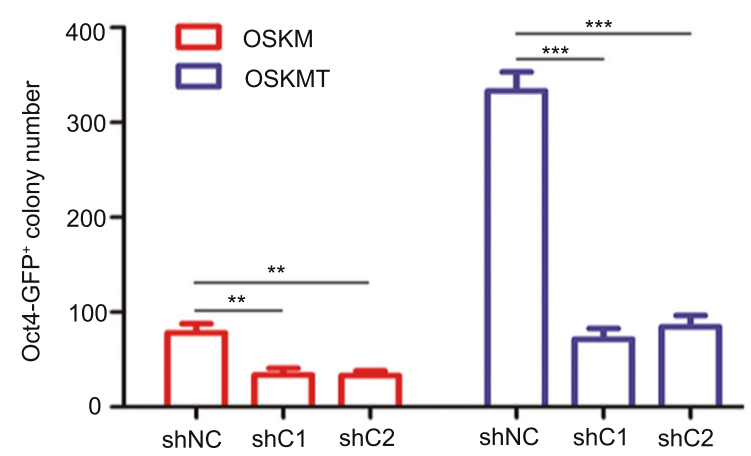

$\mathrm{H}$

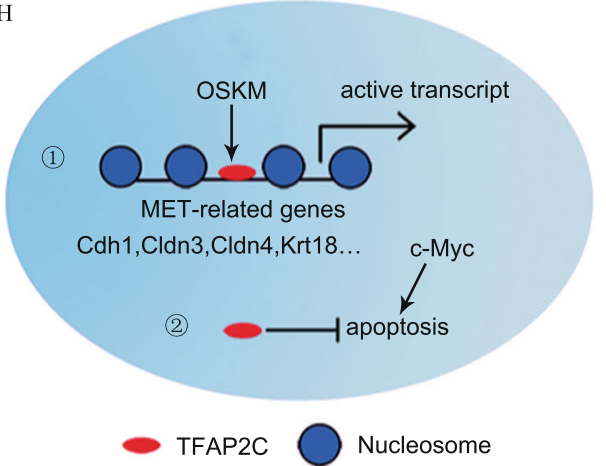

Fig. 6 (See legend on next page.) 
(see figure on previous page)

Fig. 6 Tfap2c activates MET regulators directly. a Presence of Tfap2c-binding sequence in the promoter of the indicated genes. $\mathbf{b}$ The binding of Flag-tagged Tfap2C on the Cdh1, Cldn3, Cldn4, and Krt18 loci was determined by ChIP-qPCR. Rabbit IgG was used as a control. c Schematic representation the reporter designed for determining Tfap2c binding on Cdh1 loci. NC, negative control. $\mathbf{d}$ Tfap2c enhanced the wild type (WT) Cdh1 reporter but not the mutated (Mut) ones in mESCs. Luciferase activity was detected at $36 \mathrm{~h}$ post-transfection. NC negative control. e qRT-PCR analysis for the MET genes using RNA lysates from MEFs reprogrammed with OSKMT plus control shRNA or two shRNAs for Cdh1 (shC1 and shC2) on day 4. f Western blot to test Cdh1-knockdown efficiency in mESCs. $\mathbf{g}$ Number of Oct4-GFP ${ }^{+}$colonies (day 12) in different groups. $\mathbf{h}$ A proposed working model schematically representing the main message of our work. Significance in panels $\mathbf{b}$ was determined by Unpaired two-tailed Student's $t$-test. Significance in panels $\mathbf{d}$ was determined by two-way AVOVA with Sidak's multiple comparisons test. Significance in panels $\mathbf{e}$ and $\mathbf{g}$ was determined by one-way ANOVA with Dunnett's test. Significance summary: ${ }^{* *} P \leq 0.01 ;{ }^{* * *} P \leq 0.001$. All data are presented as mean \pm S.D., $N=3$.

idea that iTSCs and iPSCs may share common transcriptional networks to establish self-renewal and may help us to better understand the internal connection between them $^{16}$. Indeed, generation of iPSCs and iTSCs both required the activation of $\mathrm{MET}$ in early stage. Given the significant role of $T f a p 2 c$ in epithelial cell identity, we speculated that Tfap $2 c$-induced MET may be a generic mechanism in Tfap $2 c$-induced reprogramming toward epithelial cells. Collectively, our results emphasize the importance of Tfap $2 c$ in regulating cell apoptosis and MET during somatic cell reprogramming and therefore provide better understanding of reprogramming mechanisms.

\section{Acknowledgements}

This study was supported by National Key R\&D Program of China grant (2017YFA0105702), the National Science and Technology Major Project (2017ZX09304023), the Science and Technology Major Project in Sichuan Province of China (2017SZDZX0012) and 1.3.5 project for disciplines of excellence in West China Hospital, Sichuan University.

\section{Author details}

${ }^{1}$ State Key Laboratory of Biotherapy and Cancer Center, West China Hospital, Sichuan University and Collaborative Innovation Center for Biotherapy, 610041 Chengdu, Sichuan, P.R. China. ${ }^{2}$ Department of Obstetrics, Sichuan Provincial Hospital for Women and Children, Chengdu, P.R. China. ${ }^{3}$ Laboratory Animal Center, Sichuan University, Chengdu, P.R. China

\section{Data availability}

All the data needed to evaluate the conclusions in the paper are present in the paper or in the Supplementary Materials.

\section{Conflict of interest}

The authors declare that they have no conflict of interest.

\section{Publisher's note}

Springer Nature remains neutral with regard to jurisdictional claims in published maps and institutional affiliations.

Supplementary Information accompanies this paper at (https://doi.org/ 10.1038/s41419-020-2684-9).

Received: 3 March 2020 Revised: 8 June 2020 Accepted: 12 June 2020 Published online: 25 June 2020

\section{References}

1. Takahashi, K. \& Yamanaka, S. Induction of pluripotent stem cells from mouse embryonic and adult fibroblast cultures by defined factors. Cell 126, 663-676 (2006).
2. Meissner, A., Wernig, M. \& Jaenisch, R. Direct reprogramming of genetically unmodified fibroblasts into pluripotent stem cells. Nat. Biotechnol. 25, 1177-1181 (2007).

3. Shi, Y., Inoue, H., Wu, J. C. \& Yamanaka, S. Induced pluripotent stem cell technology: a decade of progress. Nat. Rev. Drug Discov. 16, 115-130 (2017).

4. Hirschi, K. K., Li, S. \& Roy, K. Induced pluripotent stem cells for regenerative medicine. Annu. Rev. Biomed. Eng. 16, 277-294 (2014).

5. Matsa, E., Ahrens, J. H. \& Wu, J. C. Human induced pluripotent stem cells as a platform for personalized and precision cardiovascular medicine. Physiol. Rev. 96, 1093-1126 (2016).

6. Rowe, R. G. \& Daley, G. Q. Induced pluripotent stem cells in disease modelling and drug discovery. Nat. Rev. Genet. 20, 377-388 (2019).

7. Buganim, Y. et al. Single-cell expression analyses during cellular reprogramming reveal an early stochastic and a late hierarchic phase. Cell 150, 1209-1222 (2012).

8. Apostolou, E. \& Stadtfeld, M. Cellular trajectories and molecular mechanisms of iPSC reprogramming. Curr. Opin. Genet. Dev. 52, 77-85 (2018).

9. Pei, D., Shu, X., Gassama-Diagne, A. \& Thiery, J. P. Mesenchymal-epithelial transition in development and reprogramming. Nat. Cell Biol. 21, 44-53 (2019).

10. Schiebinger, G. et al. Optimal-transport analysis of single-cell gene expression identifies developmental trajectories in reprogramming. Cell 176, 928-943. e922 (2019).

11. Eckert, D., Buhl, S., Weber, S., Jager, R. \& Schorle, H. The AP-2 family of transcription factors. Genome Biol. 6, 246 (2005).

12. Pinto, L. et al. AP2gamma regulates basal progenitor fate in a region- and layer-specific manner in the developing cortex. Nat. Neurosci. 12, 1229-1237 (2009).

13. Qiao, Y. et al. AP2gamma regulates neural and epidermal development downstream of the BMP pathway at early stages of ectodermal patterning. Cell Res. 22, 1546-1561 (2012).

14. Krendl, C. et al. GATA2/3-TFAP2A/C transcription factor network couples human pluripotent stem cell differentiation to trophectoderm with repression of pluripotency. Proc. Natl Acad. Sci. USA 114, E9579-E9588 (2017).

15. Sharma, N. et al. Tpbpa-Cre-mediated deletion of TFAP2C leads to deregulation of Cdkn1a, Akt1 and the ERK pathway, causing placental growth arrest. Development 143, 787-798 (2016).

16. Kidder, B. L. \& Palmer, S. Examination of transcriptional networks reveals an important role for TCFAP2C, SMARCA4, and EOMES in trophoblast stem cell maintenance. Genome Res. 20, 458-472 (2010).

17. Benchetrit, $\mathrm{H}$. et al. Extensive nuclear reprogramming underlies lineage conversion into functional trophoblast stem-like cells. Cell Stem Cell 17, 543-556 (2015).

18. Chen, D. et al. The TFAP2C-regulated OCT4 naive enhancer is involved in human germline formation. Cell Rep. 25, 3591-3602. e3595 (2018).

19. Schwarz, B. A. et al. Prospective isolation of poised iPSC intermediates reveals principles of cellular reprogramming. Cell Stem Cell 23, 289-305. e285 (2018).

20. Benchetrit, $\mathrm{H}$. et al. Direct induction of the three pre-implantation blastocyst cell types from fibroblasts. Cell Stem Cell 24, 983-994. e987 (2019).

21. Pastor, W. A. et al. TFAP2C regulates transcription in human naive pluripotency by opening enhancers. Nat. Cell Biol. 20, 553-564 (2018).

22. $L i, H$. et al. RNA helicase DDX5 inhibits reprogramming to pluripotency by miRNA-based repression of RYBP and its PRC1-dependent and -independent functions. Cell Stem Cell 20, 462-477. e466 (2017).

23. Brambrink, T. et al. Sequential expression of pluripotency markers during direct reprogramming of mouse somatic cells. Cell Stem Cell 2, 151-159 (2008).

24. Chronis, C. et al. Cooperative binding of transcription factors orchestrates reprogramming. Cell 168, 442-459. e420 (2017). 
25. Vaquerizas, J. M., Kummerfeld, S. K., Teichmann, S. A. \& Luscombe, N. M. A census of human transcription factors: function, expression and evolution. Nat. Rev. Genet. 10, 252-263 (2009).

26. Kang, H. J. et al. Differential regulation of estrogen receptor alpha expression in breast cancer cells by metastasis-associated protein 1. Cancer Res. 74, 1484-1494 (2014).

27. Gingold, J. A. et al. A genome-wide RNAi screen identifies opposing functions of Snai1 and Snai2 on the Nanog dependency in reprogramming. Mol. Cell 56, 140-152 (2014).

28. Samavarchi-Tehrani, P. et al. Functional genomics reveals a BMP-driven mesenchymal-to-epithelial transition in the initiation of somatic cell reprogramming. Cell Stem Cell 7, 64-77 (2010).

29. Pieters, T. \& van Roy, F. Role of cell-cell adhesion complexes in embryonic stem cell biology. J. Cell Sci. 127, 2603-2613 (2014).

30. Sakurai, K. et al. Kinome-wide functional analysis highlights the role of cytoskeletal remodeling in somatic cell reprogramming. Cell Stem Cell 14, 523-534 (2014).

31. $\mathrm{Ma}$, T. et al. Atg5-independent autophagy regulates mitochondrial clearance and is essential for iPSC reprogramming. Nat. Cell Biol. 17, 1379-1387 (2015)

32. Gonzalez, F. \& Huangfu, D. Mechanisms underlying the formation of induced pluripotent stem cells. Wiley Interdiscip. Rev. Dev. Biol. 5, 39-65 (2016).

33. Banito, A. et al. Senescence impairs successful reprogramming to pluripotent stem cells. Genes Dev. 23, 2134-2139 (2009).

34. Li, H. et al. The Ink4/Arf locus is a barrier for iPS cell reprogramming. Nature 460, 1136-1139 (2009).

35. Kim, E. J. Y. et al. BAK/BAX-mediated apoptosis is a Myc-induced Roadblock to reprogramming. Stem Cell Rep. 10, 331-338 (2018).

36. McMahon, S. B. MYC and the control of apoptosis. Cold Spring Harb. Perspect. Med. 4, a014407 (2014).

37. Liu, X. et al. Sequential introduction of reprogramming factors reveals a timesensitive requirement for individual factors and a sequential EMT-MET mechanism for optimal reprogramming. Nat. Cell Biol. 15, 829-838 (2013).

38. Chen, T. et al. E-cadherin-mediated cell-cell contact is critical for induced pluripotent stem cell generation. Stem Cells 28, 1315-1325 (2010).

39. Li, R. et al. A mesenchymal-to-epithelial transition initiates and is required for the nuclear reprogramming of mouse fibroblasts. Cell Stem Cell 7, 51-63 (2010).

40. Lowry, W. E. E-cadherin, a new mixer in the Yamanaka cocktail. EMBO Rep. 12 613-614 (2011).

41. Redmer, T. et al. E-cadherin is crucial for embryonic stem cell pluripotency and can replace OCT4 during somatic cell reprogramming. EMBO Rep. 12, 720-726 (2011).

42. Cyr, A. R. et al. TFAP2C governs the luminal epithelial phenotype in mammary development and carcinogenesis. Oncogene 34, 436-444 (2015).
43. Marion, R. M. et al. A p53-mediated DNA damage response limits reprogramming to ensure iPS cell genomic integrity. Nature 460, 1149-1153 (2009).

44. Liu, J. et al. The oncogene c-Jun impedes somatic cell reprogramming. Nat. Cell Biol. 17, 856-867 (2015).

45. Weltner, J. et al. Human pluripotent reprogramming with CRISPR activators. Nat. Commun. 9, 2643 (2018).

46. Deng, W. et al. MicroRNA replacing oncogenic Klf4 and c-Myc for generating iPS cells via cationized Pleurotus eryngii polysaccharide-based nanotransfection. ACS Appl. Mater. Interfaces 7, 18957-18966 (2015).

47. Hu, X. et al. Tet and TDG mediate DNA demethylation essential for mesenchymal-to-epithelial transition in somatic cell reprogramming. Cell Stem Cell 14, 512-522 (2014).

48. Wu, J., Ocampo, A. \& Belmonte, J. C. I. Cellular metabolism and induced pluripotency. Cell 166, 1371-1385 (2016).

49. Liu, L., Michowski, W., Kolodziejczyk, A. \& Sicinski, P. The cell cycle in stem cell proliferation, pluripotency and differentiation. Nat. Cell Biol. 21, 1060-1067 (2019).

50. Hong, $\mathrm{H}$. et al. Suppression of induced pluripotent stem cell generation by the p53-p21 pathway. Nature 460, 1132-1135 (2009).

51. Ozmadenci, D. et al. Netrin-1 regulates somatic cell reprogramming and pluripotency maintenance. Nat. Commun. 6, 7398 (2015).

52. Zhuang, Q. et al. NCoR/SMRT co-repressors cooperate with c-MYC to create an epigenetic barrier to somatic cell reprogramming. Nat. Cell Biol. 20, 400-412 (2018).

53. Park, J. M. et al. The role of Tcfap2c in tumorigenesis and cancer growth in an activated Neu model of mammary carcinogenesis. Oncogene 34, 6105-6114 (2015).

54. Hilger-Eversheim, K., Moser, M., Schorle, H. \& Buettner, R. Regulatory roles of AP-2 transcription factors in vertebrate development, apoptosis and cell-cycle control. Gene 260, 1-12 (2000).

55. Li, L. et al. TFAP2C- and p63-dependent networks sequentially rearrange chromatin landscapes to drive human epidermal lineage commitment. Cell Stem Cell 24, 271-284. e278 (2019).

56. Wang, $X$. et al. TFAP2C promotes stemness and chemotherapeutic resistance in colorectal cancer via inactivating hippo signaling pathway. J. Exp. Clin. Cancer Res. 37, 27 (2018).

57. De Andrade, J. P. et al. EGFR is regulated by TFAP2C in luminal breast cancer and is a target for Vandetanib. Mol. Cancer Ther. 15, 503-511 (2016).

58. Kolat, D., Kaluzinska, Z., Bednarek, A. K. \& Pluciennik, E. The biological characteristics of transcription factors AP-2alpha and AP-2gamma and their importance in various types of cancers. Biosci. Rep. 39, BSR20181928 (2019). https://doi.org/10.1042/BSR20181928. 